\title{
Convergencia europea y modernización de las instituciones presupuestarias ${ }^{1}$
}

José Manuel González-Páramo*

\section{Convergencia europea y disciplina presupuestaria}

Como es bien sabido, el proceso de crecimiento de la producción de una economía tiene unos fundamentos básicamente reales y microeconómicos, que son los que determinan las decisiones de trabajo, de ahorro, de inversión y de cambio tecnológico. Sin embargo, no es posible ignorar el papel de las polítjcas macroeconómicas como condicionantes de la continuidad y de la propia posibilidad de los procesos de crecimiento y convergencia económica real. Sin estabilidad de las variables macroeconómicas nominales, los agentes económicos tienen que soportar una mayor incertidumbre, los precios relativos dejan de transmitir información correcta y los ajustes reales que la competitividad demanda se realizan con dificultad. La inestabilidad nominal tiende a elevar los tipos de interés nominales y reales, incentiva la realización de actividades directamente improductivas que persiguen la protección frente a la inflación (por ejemplo, la indiciación de los salarios y otras rentas) erosiona la rentabilidad de la inversión en los sectores de bienes comercializables y potencia los efectos distorsionantes del sistema fiscal. En suma, una inflación relativamente alta, variable y poco predecible, afecta negativamente al funcionamiento de los mercados y, por tanto, a las posibilidades de crecimiento de la economía.

La evidencia empírica disponible sobre la conexión entre la evolución de la productividad y el nivel o la aceleración de indicadores de estabilidad nominal es coincidente en sus grandes líneas: la inflación reduce el crecimiento a largo plazo de la productividad del trabajo. Así, KORMENDI y MEGURRE (1985) identifican una relación negativa y significativa entre la aceleración de la inflación y el crecimiento a largo plazo, resultado que reproducen recientemente ANDRÉs y otros (1995) para las economías de la OCDE con la tasa de inflación y la variabilidad de las tasas de crecimiento monetario. Por su parte, tanto FISCHER (1991) como Grimes (1991) encuentran una asociación negativa fuerte entre inflación y crecimiento con modelos de corte transversal y de panel. Esta última técnica ha sido la empleada recientemente por RAYMOND (1993), cuya especificación empírica postula que la inflación afecta a la eficiencia en la producción, dada una utilización de inputs. La estimación para los países de la CE en el período 1961-1991 produjo un efecto negativo, significativo y muy robusto, de la inflación sobre la tasa de crecimiento de la producción.

Esta asociación negativa entre crecimiento económico real e inflación tiene implicaciones normativas. Si la inestabilidad nominal perjudica a la competitividad y, por tanto, a las posibilidades de convergencia y crecimiento sostenido, el diseño de la mezcla de políticas macroeconómicas debería orientarse a conseguir estabilidad y baja inflación. Que este propósito sea factible depende tanto de la voluntad de las autoridades y de factores estructurales (por ejemplo, peso relativo del sector productor de bienes no comercializables), como del soporte institucional -reglas, cultura organizativa y mecanismos de decisión y supervisión- de la política macroeconómica. Sin instituciones adecuadas de política económica, las autoridades tropiezan con dificultades para promover estabilidad y para "anclar" la credibilidad de sus compromisos, en perjuicio de su potencial de crecimiento económico (WILLAMSON, 1993).

El proceso de reformas en que se está concretando la construcción de la Unión Económica y Monetaria (UEM) ofrece la mejor ilustración de la importancia creciente quc en el debate sobre política económica se asigna al diseño institucional. Así, en el ámbito de la política monetaria, el grado de independencia de los bancos centrales para desarrollar sus funciones estabilizadoras -control sobre los agregados monetarios, los tipos de interés o los tipos de cambio- a salvo de las presiones del oportunismo político de corto plazo (ciclo económico electoral) se juzga por muchos esencial para la credibilidad y la efectividad de la lucha contra la inflación.

Alesina (1988), Grilli y otros (1991), De LonG y SummerS (1992) y CuKIERMan (1992) han documentado una asociación estadística negativa muy significativa entre inflación y diversos 
indices de independencia del banco central ${ }^{2}$. De LONG y SumMERS, que estudian el período 1955-1990, asimismo obtienen un resultado empírico consistente con los comentados anteriormente: un país que decide aumentar la independencia de su banco central tiende a registrar una aceleración en la tasa de crecimiento de la productividad del trabajo. Aunque esta conexión entre independencia y crecimiento es más débil, no existe evidencia de costes en output asociados a una mayor independencia (Poliard, 1993). Ello viene a subrayar los efectos positivos asociados a una creación institucional: salvo que la independencia exacerbase el conflicto entre las políticas monetaria y fiscal, la credibilidad de los objetivos de estabilidad de la politica macroeconómica saldría beneficiada.

De igual forma, la teoría y los estudios disponibles ponen de manifiesto que tan importante es para la credibilidad de la política antiinflacionista la independencia del banco central, como la consistencia de la política presupuestaria con los objetivos de estabilidad nominal. No en vano dentro del índice de GrILL y otros (1991) juega un papel esencial la capacidad de los gobiernos para forzar al banco central a financiar los déficit públicos. Por otra parte, la indisciplina presupuestaria -entendiendo por tal la tendencia a acumular déficit insostenibles, en términos de la restricción presupuestaria intertemporal del gobierno- resta credibilidad a los objetivos de estabilidad, dado que la falta sistemática de ajuste presupuestario sólo puede conducir a la monetización o al repudio de la deuda.

A la vista de estos argumentos, no puede sorprender el énfasis que el Tratado de Maastricht pone en la disciplina presupuestaria, sin la cual la concesión de mayor independencia a los baricos centrales y la creación del Banco Central Europeo no se juzgan suficientes para garantizar la credibilidad del objetivo de convergencia nominal. El Tratado de Maastricht contiene, a este respecto, tres importantes prohibiciones, que cierran las posibilidades de monetización del déficit (art. 104), de acceso a financiación privilegiada en el sistema financiero (art. 104a) y de asunción de compromisos entre Administraciones Públicas de todos los niveles dentro de la Comunidad (art. 104b). Estas normas, vigentes desde enero de 1994, deberían ser suficientes para asegurar unas finanzas públicas saneadas. Sin embargo, en lo que es la parte más controvertida del diseño fiscal de la UEM, a ellas se añaden dos reglas: el déficit público y la deuda pública no deben exceder del 3 y el 60 por 100 del PIB, respectivamente. El incumplimiento dará lugar a la iniciación de un "procedimiento de déficit excesivo" que podría conducir a la imposición de sanciones.

Los criterios de déficit y deuda pública -cuya justificación y efectividad han dado lugar a fundadas críticas (por ejemplo, BUITER, 1992; BUITER y otros, 1992; BEAN, 1992; Gros, 1991)reflejan una doble desconfianza, puesta de manifiesto explícitamente en el Informe Delors (Comité Delors, 1989, y Comi- sión CE, 1991). Por una parte, se teme que la UEM elimine incentivos a la disciplina, al facilitar la financiación de los déficit en unos mercados de capitales más amplios. Pero quizás el motivo dominante de preocupación, respaldado por la ya referida literatura empírica sobre la economía política de los déficit públicos, es la influencia de los factores políticos e institucionales en la determinación de la politica presupuestaria. Si la estabilidad precaria de los gobiernos, la polarización ideológica, el signo político de los gobiernos en el espectro izquierda-derecha, o la ausencia de una tradición política de control del gobierno, por ejemplo, introdujesen un sesgo en favor del déficit, como estos estudios indican, los criterios de Maastricht podrían afectar al juego político favoreciendo el consenso sobre la necesidad de austeridad en los países con mayores desequilibrios.

\section{Economía, instituciones y disciplina presupuestaria}

La preocupación por la rigidez del déficit y por la aparición de situaciones de insostenibilidad del endeudamiento público a principios de la presente década ha precipitado un cierto consenso en torno a la necesidad de reducir significativamente los desequilibrios presupuestarios. Este consenso se cimenta en tres principios generales. Primero, la inestabilidad nominal erosiona las posibilidades de convergencia real a través del crecimiento económico sostenido. La mezcla de políticas fiscal y monetaria debe ser, en consecuencia, equilibrada, sostenible y disciplinada. Sólo así es posible ofrecer a los agentes económicos un marco macroeconómico de estabilidad nominal e inflación reducida. Segundo, el éxito en materia de disciplina depende tanto de la voluntad política de las autoridades como de la existencia de diseños institucionales apropiados. La consecución de buenos resultados macroeconómicos es difícil cuando las instituciones monetarias y fiscales configuran incentivos y técnicas de resolución de conflictos que resultan inconsistentes con la disciplina. Tercero, el gasto público es la variable llamada a protagonizar los procesos de consolidación presupuestaria ${ }^{3}$.

Pese a la coincidencia en las grandes líneas de esta filosofia, los resultados de las políticas de consolidación presupuestaria han sido muy desiguales. No es tarea fácil identificar las causas, aunque este conocimiento sea esencial para el diseño de estrategias conducentes a la disciplina fiscal en casos concretos, como sería el de España. Los estudios más solventes ${ }^{4}$ sobre el comportamiento de las finanzas públicas en los países industrializados permiten explicar la heterogénea evolución de los déficit públicos en términos de dos clases de factores: económicos y político-institucionales. Diferencias en el grado de desarrollo, la tasa de paro, el crecimiento económico, la evolución 
de los tipos de interés o la inflación determinan los cambios observados en la posición financiera de los gobiernos, a través de los efectos de estas variables económicas sobre distintas partidas de gastos e ingresos públicos.

Pero tanto o más importante que este conjunto de factores es el papel que juegan los diseños institucionales y los rasgos del sistema político en cuyo seno se desarrollan los procesos de formación de la política presupuestaria. Entre estos rasgos se cuentan: 1) La legislación electoral, que determina tanto la frecuencia de los cambios de gobierno como la posibilidad de formar gobiernos mayoritarios, de minoría o de coalición. Las legislaturas breves y los gobiernos de coalición suelen acompañarse de una mayor laxitud presupuestaria; 2) El grado de polarización ideológica, asociado o no a la desigualdad redistributiva. La polarización de las preferencias políticas puede retrasar los procesos de saneamiento, así como estimular la utilización de la deuda como variable estratégica; 3) La legislación constitucional en materia presupuestaria, en la medida en que constriñe la actuación de los gobiernos durante cada legislatura. El papel de esta legislación puede ser tanto más relevante cuanto más acentuado sea el comportamiento oportunista de los gobernantes en un mercado político dominado por la ilusión fiscal; 4) El grado de independencia del banco emisor, determinante de las posibilidades de acceso del gobierno a la monetización de la deuda; 5) Los procedimientos presupuestarios que rigen el diseño, el debate, la aprobación, la ejecución y el control del presupuesto.

La complejidad de los fenómenos presupuestarios -cuya interconexión se refleja esquemáticamente en la figura 1-y sus peculiaridades idiosincráticas invalidan toda pretensión de definir una estrategia única y superior a las restantes para garantizar la disciplina presupuestaria en todos los países 5 . Antes bien al contrario: la convergencia en materia de disciplina fiscal probablemente requiera la aplicación de estrategias diversas de reforma institucional. Sin embargo, el análisis de la situación de las

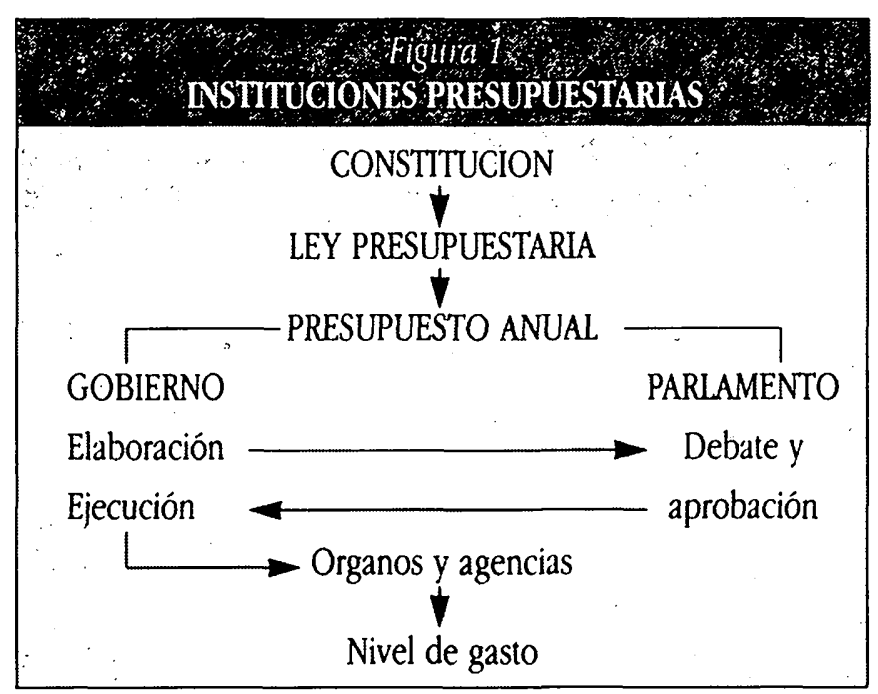

finanzas públicas en un país puede beneficiarse del examen de las grandes opciones que ofrece la experiencia comparada: la reforma de los procesos presupuestarios, el gerencialismo y la limitación constitucional. De esta tarea se ocupan brevemente las secciones siguientes ${ }^{6}$.

\section{Economía política del presupuesto y disciplina fiscal}

La institución presupuestaria es tan importante, como reflejo de las elecciones colectivas, como compleja, debido a la variedad de funciones que tiene atribuidas. No es posible evaluar el sesgo hacia la disciplina o la laxitud que está implícito en el presupuesto sin atender a la forma concreta en que estas funciones impregnan el documento presupuestario y el proceso de elaboración, discusión, ejecución y control. Las funciones más tradicionales del presupuesto son las que podríamos llamar administrativas. Estas se concretan en tres propósitos diferentes: previsión, planificación y control. El presupuesto debe ofrecer a los agentes económicos, privados y públicos, previsiones de calidad para la toma de decisiones. Como instrumento de planificación, debe asegurar la consistencia de los gastos y el déficit con las variables macroeconómicas básicas a medio y largo plazos. El presupuesto debe ser, asimismo, un medio transparente de rendición de cuentas y de exigencia de responsabilidad sobre el grado de cumplimiento del mandato recibido del legislativo.

A las funciones administrativas se añadieron, desde mediados del presente siglo, las estabilizadoras: el presupuesto es un instrumento fundamental para contrarrestar las oscilaciones cíclicas de la economía. Aunque la experiencia de los años setenta hace hoy contemplar con gran escepticismo el componente más activista de estas funciones, los estabilizadores automáticos (por ejemplo, los impuestos sobre la renta o las prestaciones por desempleo) permanecen como elementos centrales en cualquier presupuesto. La creación de la UEM vendrá a realzar la importancia del papel estabilizador del presupuesto, al quedar convertido en el único instrumento de política macroeconómica capaz de contrarrestar perturbaciones específicas sufridas por cada país, una vez perdido el control de los gobiernos nacionales sobre el tipo de cambio y sobre la política monetaria.

El proceso presupuestario es, asimismo, un punto focal en la resolución de conflictos entre los agentes participantes: ministros del gobierno, legisladores y ciudadanos. En sentido amplio, el proceso presupuestario es un conjunto de reglas formales -constitución, leyes, decretos y órdenes-e informales 
-prácticas y normas de comportamiento de la cultura presupuestaria- que establecen el papel institucional de los agentes, la secuencia de las decisiones y el procedimiento para alcanzar compromisos. Estas reglas son la esencia del proceso y determinan sus resultados, al asignar poderes de decisión y papeles estratégicos, determinar los flujos de información, crear o destruir oportunidades de colusión entre agentes y regular la transparencia de las decisiones y la responsabilidad sobre su adecuada ejecución (VON HAGEN y HARDEN, 1994). Un buen proceso presupuestario es aquel que facilita una resolución efectiva de conflictos, generando acuerdos estables y predecibles entre legisladores y gobernantes e incentivando el uso eficiente de los recursos públicos. Esta es la función politico-económica del presupuesto.

Existen tres fuentes fundamentales de ineficiencia que aquejan a los procesos presupuestarios, y que tienen como consecuencia una escasa disciplina y un nivel de gasto excesivo: 1) Existencia de metas políticas particulares (prestigio, promoción política, etc.) de algunos participantes en el proceso, que promueven el uso de los fondos públicos para fines poco relacionados con el interés colectivo; 2) Ilusión fiscal, es decir, el sesgo hacia un gasto excesivo cuando un agente con poder de decisión en materia de gasto no considera el verdadero coste de financiarlo, al desplazarse en parte a otros agentes o quedar éste diluido entre el conjunto de los contribuyentes, y 3) Propensión al déficit, que emerge cuando los intereses de los futuros contribuyentes no están adecuadamente representados en las decisiones de gasto adoptadas en el presente. Estos rasgos pueden influir con intensidad variable en las distintas fases del proceso presupuestario.

En el proceso de elaboración del presupuesto, los ministros de gasto (MG) tienen un interés natural en expandir sus actividades, ya que la financiación del gasto se distribuye entre la ciudadanía y el éxito político se mide en términos del beneficio neto de sus clientelas respectivas. Si los ministros MG pudieran elaborar sus propuestas de gasto sin restricciones, el universalismo (el presupuesto debe contener algo para todos) y la reciprocidad (intercambio de apoyos para programas no excluyentes) -expresión de las metas políticas particulares- consolidarian la ilusión fiscal y, por tanto, un déficit excesivo. Al mismo tiempo, en los gabinetes ministeriales miembros cuyas decisiones responden más al interés colectivo del gobierno (MC): presidente, vicepresidentes o ministros sin cartera y el ministro de Hacienda. Un proceso presupuestario que refuerza el papel de estos últimos en la elaboración limitará la incidencia de la ilusión fiscal.

Hay dos "tecnologías de compromiso" básicas para lograr la prevalencia del interés colectivo dentro del gobierno (VON HAGEN y HARDEN, 1994). La primera consiste en asignar una posición de dominio estratégico a los miembros MC del gobierno, que actúan como directores del proceso, monopolizan la información esencial, tienen autoridad para conseguir acuerdos bilaterales entre ministros $M G$, preparan la agenda de las decisiones del gabinete o tienen poder de veto. Un modo alternativo de proceder sería que los miembros MC determinasen los objetivos cuantitativos sobre el volumen y otros parámetros básicos del presupuesto, de manera que se disciplinase de forma estricta el universalismo y la reciprocidad en las fases ulteriores de discusión y ejecución?

Por otra parte, puesto que los ministros MG tienden a obtener más ventajas políticas derivadas del apoyo de los beneficiarios de los gastos corrientes y su carrera política es con frecuencia más corta que la de los miembros $\mathrm{MC}$, los procesos que refuerzan el papel de estos últimos también reducen la laxitud debida al sesgo deficitario. El compromiso del gobierno con una serie de objetivos presupuestarios plurianuales es la forma más frecuente en que este principio se hace operativo.

Tras la elaboración, el presupuesto entra en el parlamento. En esta fase del proceso vuelven a emerger el mismo conflicto de intereses particulares y generales y análoga tendencia al universalismo y la reciprocidad. El resultado de la discusión y la aprobación en el parlamento depende básicamente de dos rasgos institucionales o procedimentales: relación de poderes entre el gobierno y el parlamento, por un lado, y entre cámara baja y cámara alta cuando ésta existe, por otro. Los procesos presupuestarios que asignan un fuerte peso estratégico al gobierno -limitando estrictamente las enmiendas, introduciendo costes políticos importantes asociados al rechazo del proyecto por el parlamento, forzando votaciones partida a partida antes de un voto global sobre el presupuesto- y a la cámara baja sobre la alta -en la que tienden a estar sobrerrepresentados intereses regionales específicos- reducen la influencia negativa sobre la disciplina de la ilusión fiscal, los intereses particulares y el sesgo deficitario.

Para que el presupuesto sea un buen instrumento de resolución de conflictos su contenido debe afectar a todos los problemas de trascendencia presupuestaria y sus normas de procedimiento han de evitar las "no decisiones". Ejemplos comunes que pugnan con este principio general son la huida del presupuesto hacia agencias u otras entidades, la concesión generosa de la categoría de automáticamente ampliables a un número excesivo de gastos, la aceptación pasiva de presupuestos anteriores como base de discusión o la conversión del presupuesto en un registro de compromisos previos. El presupuesto no debe poner en cuestión los derechos subjetivos que reconozcan las leyes, pero sí decidir sobre niveles de cobertura, prestación o retribución y, en todo caso, debe ofrecer la mayor riqueza posible de información sobre estos compromisos y sobre actividades extrapresupuestarias y obligaciones contingentes.

Durante la ejecución del presupuesto bajo control del gobierno los ministros MG tienen más incentivos que los 


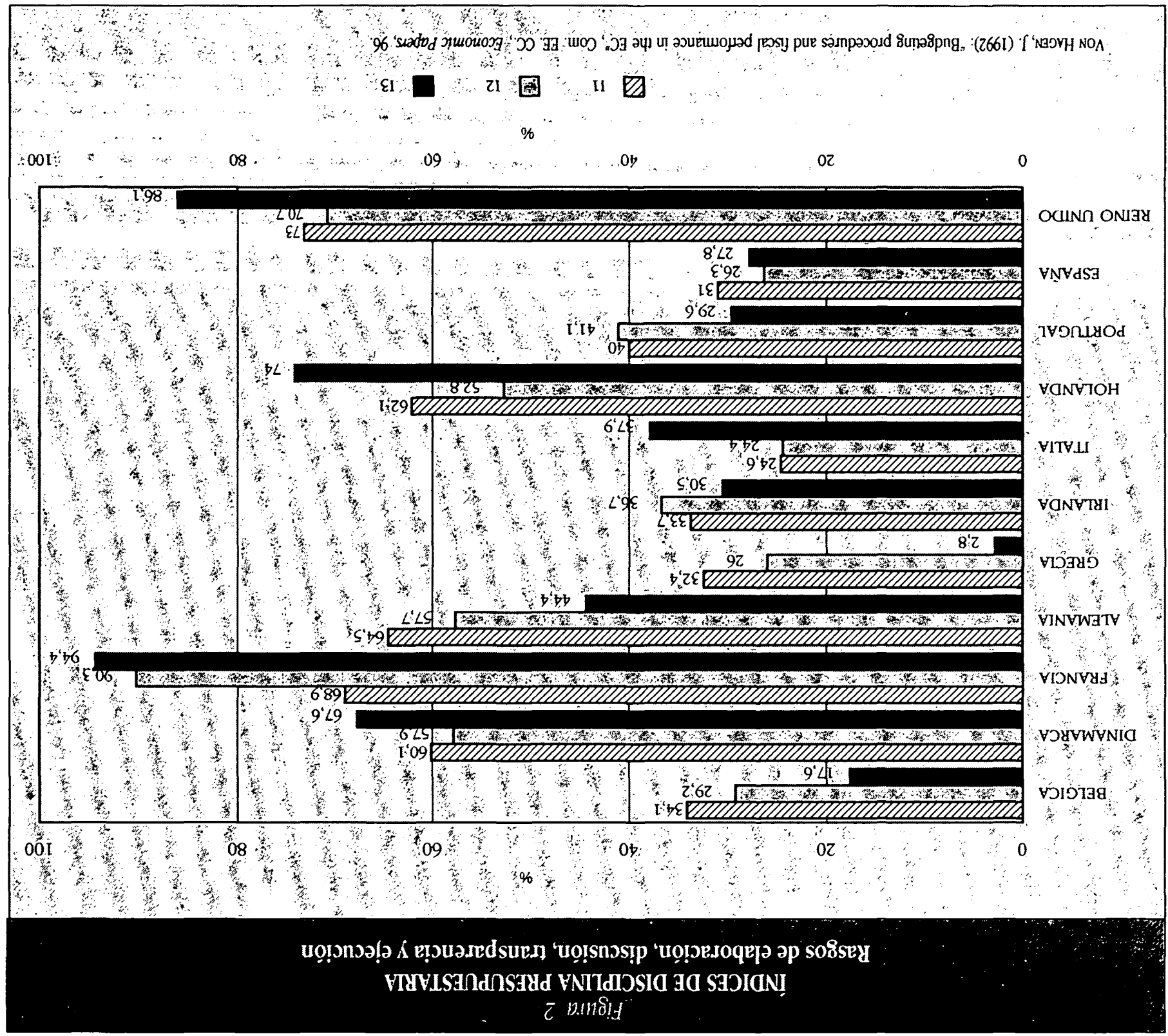

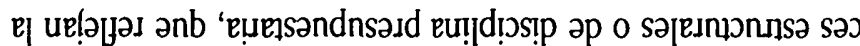

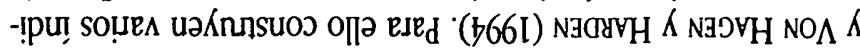

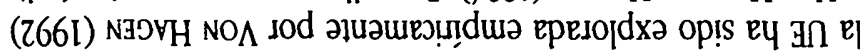

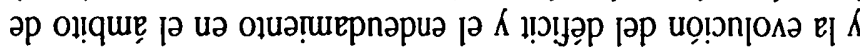

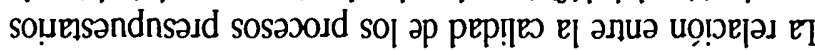

sopuoj $ә \mathrm{p}$

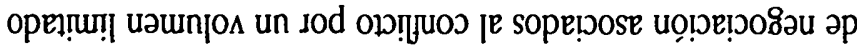

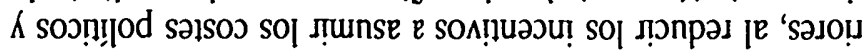

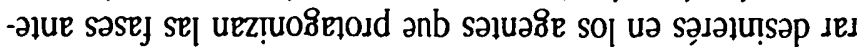

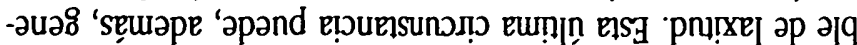

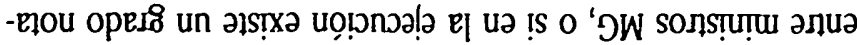

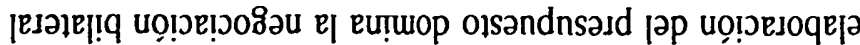

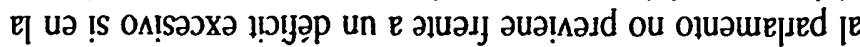

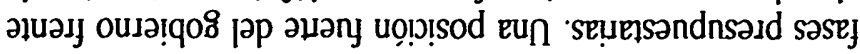

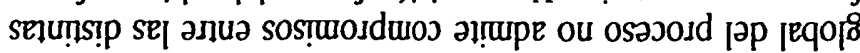

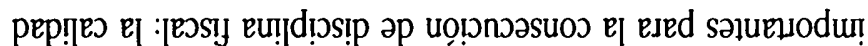

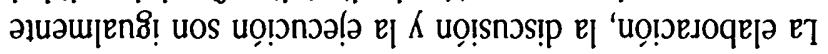

ग|qе!|dure

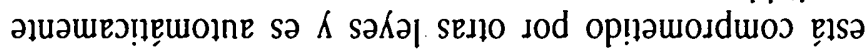
olse8 sol $e$ olse8 ap sədol sauodun esed sezueu!y әр әqqesuodsas

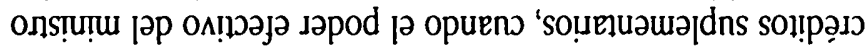
әр uọ़uəıqо в

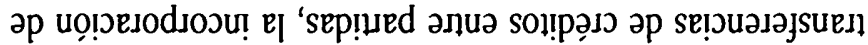

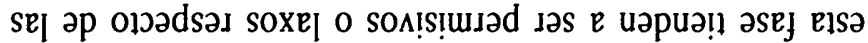

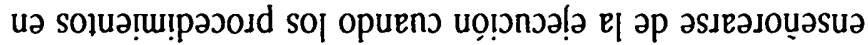

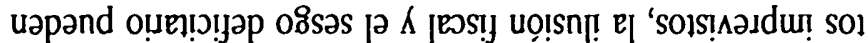

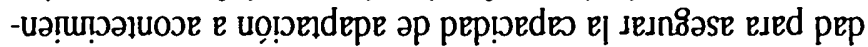

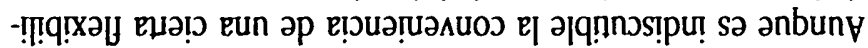

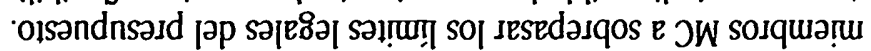


medida en que las características de los procesos nacionales tienden a reducir la influencia de la ilusión fiscal. La figura 2 representa estos índices: I1 refleja la naturaleza de los procesos de elaboración, discusión y ejecución, así como el carácter informativo del proyecto presupuestario; 12 excluye del anterior los aspectos de información, e I3 sólo contempla las fases de elaboración y debate parlamentario ${ }^{8}$.

La evidencia analizada por estos autores ofrece un fuerte respaldo a la bipótesis de ilusión fiscal: las características institucionales que refuerzan el papel estratégico del primer ministro y de los restantes miembros MC del gobierno en relación a los ministros $M G$, que se adhieren a objetivos presupuestarios cuantitativos estrictos al comienzo del proceso, que afirman la posición negociadora del gobierno frente al parlamento -limitando las posibilidades de enmienda y exigiendo votaciones por partidas y un voto global sobre el volumen total del presupuesto en la primera fase de la discusión-, que resaltan el carácter informativo del proyecto y que limitan rígidamente la flexibilidad en la ejecución, tienden a conducir a la disciplina fiscal, medida en términos de menores ratios de gasto, déficit y deuda. De HaAn y Sturm (1994) han analizado la robustez de este resultado empírico una vez se añaden como variables explicativas otros determinantes económicos (tasa de paro, tipo de interés de la deuda y crecimiento de la población) y políticos (frecuencia de los cambios en el gobierno). Sus resultados refuerzan el respaldo a la hipótesis de ilusión fiscal. Por su parte, la bipótesis de sesgo deficitario, según la cual la disciplina fiscal se beneficia de la existencia de un fuerte compromiso político con objetivos presupuestarios plurianuales, una gran calidad informativa del proyecto y unas fuertes limitaciones a las enmiendas parlamentarias y a la flexibilidad en la ejecución, no obtiene un apoyo tan nítido: estos rasgos contribuyen a la disciplina, pero lo hacen de forma limitada.

Estas sugerentes conclusiones parecen, sin duda, respaldar la necesidad de revisar los procedimientos presupuestarios en los países más laxos, entre los que se cuenta España, si éstos han de lograr éxitos en el objetivo de disciplinar sus finanzas públicas. Sin embargo, puesto que el presupuesto tiene más funciones que las político-económicas, cabría inquirir acerca de los posibles costes de una mayor disciplina. VON HAGEN y HaRDEN (1994) se ocupan también de esta cuestión. Sus resultados son confortantes, ya que sugieren que las complementariedades entre las funciones político-económica, administrativa y estabilizadora del presupuesto dominan sobre los posibles conflictos. En concreto, la evidencia indica que aquellos países con instituciones y procedimientos más disciplinados: 1) Tienen presupuestos con mayor calidad para la previsión y el control; 2) No pierden su capacidad de respuesta para contrarrestar las oscilaciones cíclicas de la economía, y lo hacen generando menores déficit, y 3) Tienden a enfrentarse a menores problemas de sostenibilidad a medio y largo plazo?.

\section{Otras opciones: del gerencialismo a la limitación constitucional}

En la sección precedente hemos analizado algunas reformas institucionales dirigidas a mejorar las reglas de coordinación y motivación de los agentes presupuestarios para la consecución de disciplina, dados el marco constitucional de la política presupuestaria y los procedimientos de gestión del gasto. La experiencia internacional ofrece también ejemplos recientes de reformas constitucionales y de los mecanismos de gestión con idéntico fin. A ellas nos referimos brevemente a continuación.

Comenzando por las reformas en los mecanismos de gestión, hoy se acepta con generalidad que una de las causas del gasto excesivo que tienden a realizar los gobiernos es la ausencia de incentivos apropiados, tanto organizativos (limitadas referencias competitivas, inexistencia de pago directo por el usuario e imposibilidad de quiebra) como individuales (escaso nexo retribución-esfuerzo y permanencia en el empleo). Desde comienzos de los años 80 , coincidiendo con una renovada confianza en las fuerzas del mercado y bajo la presión de la necesidad de sanear las finanzas públicas, se ha producido una oleada de cambios dirigidos a mejorar la relación coste-eficacia en las actividades públicas. El gerencialismo en el sector público ha venido a acentuar cada vez más la orientación al usuario y la consecución de resultados cuantificados. Estas reformas incluyen la introducción paulatina de flexibilidad en la contratación o el empleo, la creación de ambientes competitivos para que las unidades administrativas contrasten sus resultados con otras unidades públicas o privadas, la separación entre producción de servicios y financiación de los mismos, el establecimiento de incentivos ligados a responsabilidades y, en todos los casos, un cambio de cultura más o menos pronunciado ${ }^{10}$.

Entre los países que han optado decididamente por esta vía de disciplina, Nueva Zelanda es el que ha introducido las reformas de modo más radical y sistemático". En 1987, tras un trienio de intensa desregulación y liberalizacion de la economía, nueve grandes departamentos ministeriales encargados de ofrecer servicios comerciales fueron convertidos en corporaciones públicas (entre ellos se cuentan el suministro de electricidad, las telecomunicaciones, los servicios postales, la gestión de bosques, la producción de carbón y el transporte aéreo). Al mismo tiempo, se eliminaron obstáculos a la entrada y las empresas públicas quedaron sometidas a la legislación comercial general. Los directores tienen la misión de gestionar con criterios lucrativos, bajo la supervisión de órganos rectores procedentes del sector privado.

El alcance de los cambios fue más radical en el sector público administrativo, al que se ha aplicado con generalidad la 
separación entre la provisión de servicios (regulación y financiación), que realiza el gobierno, y su producción, que es realizada bien por una agencia pública -en competencia con otras agencias públicas o con empresas privadas, bajo control del ministro correspondiente y del Tesoro público- o bien directamente por empresas privadas. Los ministros son compradores de servicios y responden de los resultados de su política ante el gobierno y el parlamento. Las agencias venden a los ministerios bienes y servicios (outputs), que producen con el capital y el presupuesto puesto a su disposición (inputs). Los responsables de las agencias son jefes ejecutivos, vinculados a los ministerios mediante contratos -con vigencia de entre dos y cinco añosen los que se especifican los objetivos de output, el rendimiento financiero y otros aspectos de la relación con la autoridad política $^{12}$. Los jefes ejecutivos tienen poder de contratación, despido y fijación de salarios, así como de comprar bienes y servicios en el sector privado o a otras agencias centrales o locales. En muchos casos, la creación de ambientes de mercado se completa con el cobro al usuario de precios o tiques moderadores.

Un sistema basado en la competencia y en la responsabilidad sobre resultados y outputs (accountability) necesita disponer de indicadores de eficacia y de instrumentos contables apropiados. De hecho, Nueva Zelanda ha introducido una de las reformas más rigurosas y de más amplio alcance del mundo en contabilidad pública. Se ha abandonado el criterio de caja por uno de gestión, que incluye de forma separada operaciones corrientes y de capital, cuentas de ingresos y gastos, balance completo (con valoración de intangibles y de obligaciones contingentes), flujos de caja y rendimientos del capital, cuyo coste de mantenimiento debe quedar reflejado. Este soporte contable permite al gobierno y al parlamento estudiar rendimientos, comparar objetivos y resultados y, por tanto, mejorar la exigencia de responsabilidad.

Pese a su corta experiencia, el radicalismo gerencial sin duda ha aumentado la transparencia, ha producido un ahorro apreciable de ineficiencia y ha disminuido al mínimo las actividades duplicadas e innecesarias. Pero la reforma también ha reducido la actividad y el esfuerzo en las dimensiones menos verificables o medibles del servicio, y ha aumentado quizás excesivamente la rotación y la movilidad de los empleados públicos, determinando cierta pérdida de memoria institucional. Tras las elecciones de 1993, que supusieron una importante pérdida de apoyo del Partido Nacional en el poder, se ha retornado a un cauto incrementalismo. Este retroceso, sin embargo, no puede interpretarse como una descalificación de la nueva gestión pública, que en grandes líneas respalda la población, sino más bien como un aval de las estrategias de reforma basadas en la introducción de cambios más selectivos, de gran alcance pero concentrados en ámbitos reducidos.

Si los procedimientos de gestión son el nivel básico de regulación de la actividad pública, en el nivel supremo tenemos las normas de rango constitucional. Siguiendo a la aprobación de la proposición 13 tras la revuelta fiscal de California, los EE.UU. se han constituido en el laboratorio por excelencia de las limitaciones constitucionales o legales a la actividad del gobierno. La defensa de los límites constitucionales sobre el déficit apela a la necesidad de poner techo a la irresponsabilidad fiscal que tiende a generar la actividad de los políticos, quienes verían en el gasto financiado con deuda un medio de ganar elecciones sin enfrentarse a una resistencia ciudadana apreciable por efecto de la ilusión fiscal, que lleva a los contribuyentes a subestimar los costes impositivos futuros (BUCHANAN y WAGNER, 1977). "No son sólo los muy malvados funcionarios y sus protectores políticos -escribe WILDAVSKY-, sino que somos todos, como dice la canción, 'you and me, babe', quienes nos creamos nuestros propios problemas" (WILDAVSKY, 1986). Problemas que se agudizan en presencia de gobiernos inestables, de polarización ideológica, o de ausencia de una tradición política de control del gobierno, y que introducen un sesgo en favor del déficit. Las limitaciones constitucionales podrían así ser contempladas como restricciones que actúan sobre el juego político, favoreciendo el consenso en materia de responsabilidad fiscal.

Las limitaciones constitucionales han sido objeto de un alud de justificadas críticas (véase FuENTES QUINTANA, 1985, y BAYOUMI y EICHENGREEN, 1995). Su cuantificación es difícil y arbitraria, las cláusulas de escape pueden hacerlas inoperantes, las sanciones por incumplimiento con frecuencia son imprecisas y poco creíbles, reducen la capacidad estabilizadora automática del presupuesto, etcétera. De entre las objeciones más sustantivas pueden destacarse dos fundamentales. Primera, las limitaciones se dirigen a los meros sintomas y no a la raíz del problema. Segun$\mathrm{da}$, su efectividad es muy dudosa. VON HAGEN ha analizado la eficacia de las limitaciones sobre deuda y déficit que hoy existen en 35 y 41 Estados norteamericanos, respectivamente ${ }^{13}$. La experiencia de estas limitaciones indica que se utiliza una infinidad de procedimientos para hurtar actividades al presupuesto, entre los que sobresale el de delegar funciones y capacidad de endeudamiento en agencias extrapresupuestarias y gobiernos locales (VON HaGen, 1991). Como ha escrito, con humor, Samuelson: "Suponga que es usted un político tipo. Le frustran los déficit. Éstos limitan el gasto que sus votantes merecen. Hay una presión constante para aumentar los impuestos. Su trabajo no es nada divertido. ¿Qué hace usted? Innova. Recurre a regulaciones, avales, empresas públicas (aunque nominalmente 'privadas') creadas para la ocasión -cualquier mecanismo que permita gastar fuera de los enloquecedores confines del presupuesto oficial-" (SAMUELSON, 1988).

Los criterios de disciplina incorporados al artículo $104 \mathrm{~b}$ del Tratado de Maastricht guardan una cierta similitud con las limitaciones constitucionales, al establecer techos cuantitativos al déficit y la deuda de los Estados miembros de la UE, y prohibir la financiación monetaria o en condiciones privilegiadas, así 
como la asunción de deudas entre administraciones. Conviene, sin embargo, señalar dos diferencias importantes. Los límites sobre déficit excesivos son interpretativos y discrecionales, fruto de la supervisión mutua. Pero lo que es quizás más relevante, el artículo 3 del Protocolo sobre Déficit Excesivo requiere que los Estados miembros adapten sus procedimientos presupuestarios si ello fuera necesario para evitar la aparición de déficit excesivos. Este mandato viene a reconocer, más allá de los síntomas que se reflejan en el déficit, la importancia de las instituciones y los procedimientos presupuestarios como causas o determinantes de la disciplina fiscal ${ }^{14}$. La consecución de objetivos de disciplina podría exigir reformas en las legislaciones presupuestarias nacionales como las apuntadas en la sección 30 incluso la creación de nuevas instituciones que refuercen la credibilidad de la política presupuestaria. En este sentido, VoN HAGEN y HaRDEN (1994) han propuesto la creación de un Consejo Nacional de la Deuda independiente del gobierno, que tendría la función de establecer, dentro de las líneas generales de la política económica del ejecutivo, el techo máximo de endeudamiento anual que puede asumir el presupuesto aprobado por el parlamento. Un mecanismo de delegación de este tipo podría conseguir mejores resultados que la rígida limitación constitucional, sin los inconvenientes que plantea toda modificación de la norma fundamental.

\section{El modelo español: una valoración}

Nuestro país ha asistido en los últimos años a un vivo debate acerca de las vías más adecuadas para disciplinar las finanzas públicas. Una valoración cabal de esta cuestión no puede abordarse haciendo abstracción del contexto político y económico en el que las instituciones presupuestarias se han generado en el pasado y se desenvuelven en la actualidad's. Desde mediados de los años 70 hasta principios de la presente década, con una breve interrupción en los años 1986 y 1987, el gasto público creció en España con una extraordinaria rapidez partiendo de una base muy pequeña. Esta evolución del sector público, fruto de una decisión política deliberada, desarrolló una cultura de aumento del gasto que legitimaba tanto el incrementalismo como la falta de correspondencia entre los planes presupuestarios y la posterior ejecución oficial.

Así, pese a los intentos más o menos decididos de integrar la elaboración del presupuesto que tienen lugar desde 1986 -creación de grupos de trabajo y, posteriormente, de la comisión funcional y de las comisiones de análisis de programas-, esta cultura de gasto creciente ha debilitado los incentivos para diseñar y consolidar mecanismos efectivos y generalmente aceptados de resolución de conflictos, ha impedido el desarro- llo de las funciones administrativas del presupuesto -previsión, planificación y control- y ha obstaculizado el uso apropiado de la política fiscal como instrumento de estabilización macroeconómica. Puede afirmarse, sin exageración, que la experiencia presupuestaria de las dos últimas décadas ha consolidado una concepción del presupuesto que hace de éste un documento básicamente declarativo, con limitada fuerza para obligar a sus gestores y escasas pretensiones de realismo.

La pesada herencia del pasado más reciente permite extraer una lección fundamental: la voluntad política no es fuerza suficiente para garantizar una consolidación presupuestaria duradera cuando las instituciones y los procedimientos presupuestarios están sesgados al déficit y promueven la ilusión fiscal. Abundando en este último punto, es un hecho bien conocido -y respaldado en los índices de la figura 2- que España tiene reglas y procedimientos presupuestarios que se encuentran entre los más laxos de la UE, junto con Bélgica, Italia y Grecia. La figura 3 representa la posición relativa de España respecto de la media de los países de la UE y del país más disciplinado en cada una de las dimensiones de los índices elaborados por VON HAGEN y HARDEN: estructura de la negociación dentro del gobierno, reglas de discusión y aprobación en el parlamento, carácter informativo del proyecto, flexibilidad en la ejecución y papel de la restricción plurianual. Como se puede apreciar con claridad, en todas las características relevantes España está alejada no ya de las mejores prácticas, sino de la media europea calculada con inclusión de nuestro país.

En la fase de elaboración, el enorme peso de los gastos comprometidos -cercanos al 80 por 100 del total, según el Informe Económico Financiero de los presupuestos para 1995-, los elevados costes de negociación entre gestores, la relativamente débil posición estratégica del ministro de Hacienda y el fracaso del intento de introducir la presupuestación por programas (que sólo se presentan a efectos informativos), impiden aspirar a una dirección estratégica del presupuesto por parte del ejecutivo, basada en objetivos, indicadores y técnicas de análisis. Por otra parte, la información presupuestaria es, aunque abundante, escasamente transparente y poco útil como instrumento de gestión eficiente: no facilita comparaciones relevantes para evaluar su contenido, ni informa sobre compromisos futuros u obligaciones contingentes, ni hasta recientemente ha permitido identificar con rapidez desviaciones que, con frecuencia, han dado lugar a la acumulación de deudas hasta que su volumen hacía inevitable la decisión política de regularizarlas.

La ejecución presupuestaria de 1993 ofrece la mejor ilustración de las consecuencias de este modelo, basado en el conflicto entre una presupuestación irreal -objetivos de déficit artificialmente bajos y confianza excesiva en el efecto del crecimiento económico- y la pretensión de poner coto a la laxi- 


\section{Figira 3 DLIENSIONES DE LA DISCIPLNA: ESPANA-UE Rasgos del proceso presupuestario en España: aspectos comparados}

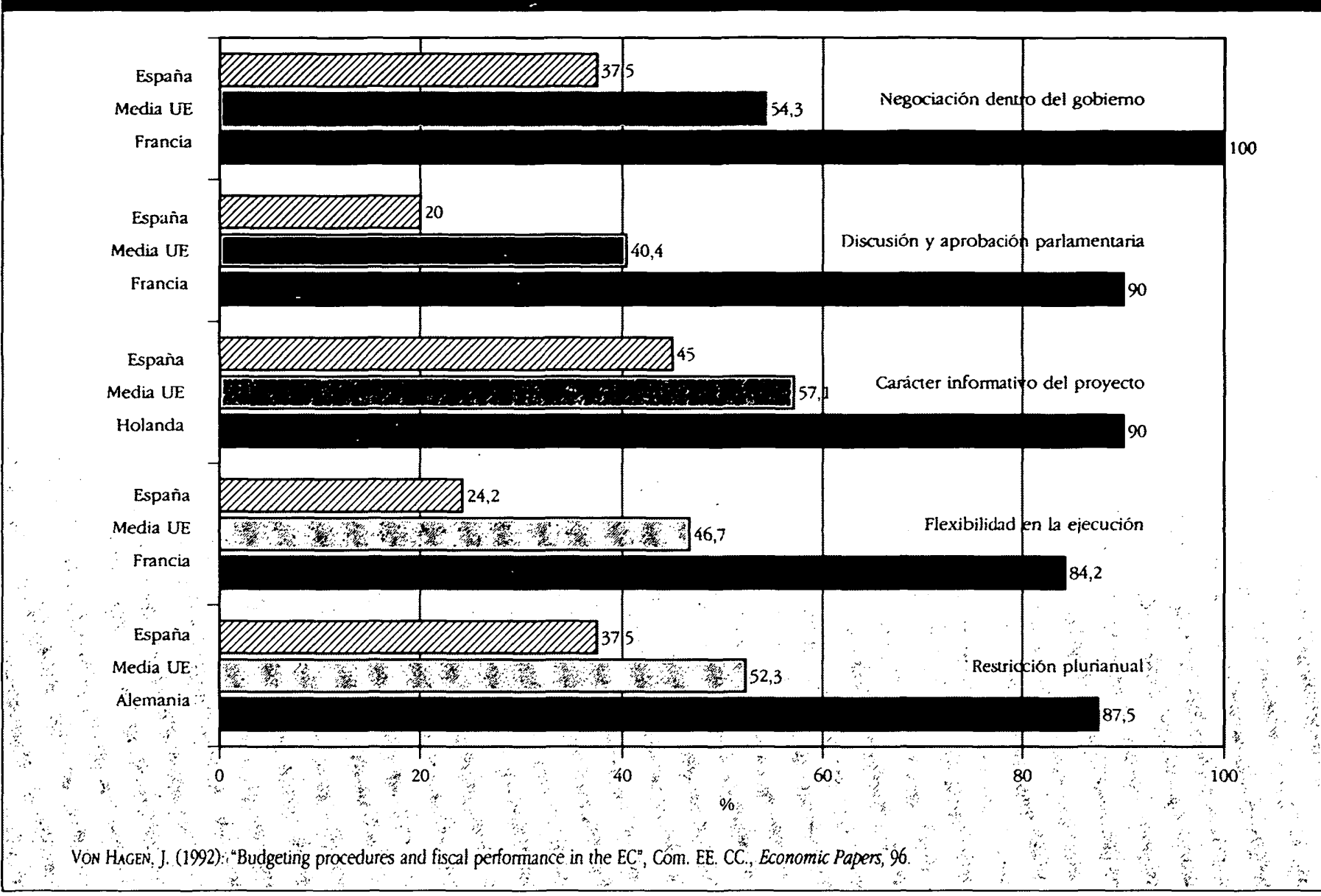

tud mediante un límite global sobre el gasto (art. 10 de la ley de presupuestos: créditos iniciales más créditos generados por ingresos, suplementarios y extraordinarios, estando estos últimos limitados en porcentajes del 2,5 por 100 en 1990,5 por 100 en 1991 y 3 por 100 en 1992 y 1993). En un marco de determinación incremental del gasto y de rápido crecimiento de éste, las desviaciones presupuestarias en la ejecución se han contrarrestado tradicionalmente de tres formas: mediante medidas extraordinarias de recorte de gastos (especialmente concentrados en las inversiones públicas) y aumentos de impuestos a mitad de ejercicio ${ }^{16}$, a través de trasvases de partidas que han venido a premiar a los malos gestores frente a los cumplidores del mandato presupuestario (las modificaciones de créditos alcanzaron un promedio del 25 por 100 respecto del presupuesto inicial entre 1983 y 1993, concentrándose en las ampliaciones de crédito y, en menor medida, en las incorporaciones de crédito del ejercicio inmediato anterior ${ }^{17}$ ) y, finalmente, mediante la interpretación elástica del principio de legalidad, realizándose gastos sin reconocimiento presupuestario. Este modelo de laxitud y aceptación política de las desviaciones presupuesta- rias hace crisis en 1993, año en el que el límite sobre gastos hubo de ser suspendido ante la magnitud del desbordamiento: la liquidación de 1993 cifraba las obligaciones reconocidas para el ejercicio en un monto que superaba en un 19 por 100 el total de los créditos iniciales, y los créditos extraordinarios y suplementarios rebasaron su límite legal en 4,6 puntos. Con este precedente, sin embargo, los presupuestos de 1994 y 1995 volvían a anclar la credibilidad de los propósitos de disciplina manteniendo el artículo 10 (art. 11 en 1995) en los mismos términos del año anterior mientras estuvo vigente ${ }^{18}$.

El problema de la credibilidad del programa de consolidación fiscal sigue siendo central para el éxito del segundo Plan de Convergencia. Puesto que la capacidad de compromiso es limitada y la reputación de disciplina fiscal es inexistente, a causa de la mala calidad relativa de los procedimientos presupuestarios, la credibilidad del segundo Plan de Convergencia sólo puede asentarse en dos pilares: un diagnóstico correcto de los problemas fundamentales y la adopción temprana de medidas de ajuste que alejen los riesgos de repetición del fracaso del primer Plan de Convergencia. A grandes rasgos, el diagnóstico 
que se contiene en el Plan parece correcto, particularmente en el énfasis en la necesidad de reducir gastos y en el reconocimiento de la naturaleza estructural de los problemas del sector público. En el detalle, no obstante, los compromisos son muy genéricos, sin plazos y, en las grandes cifras, sustentados en la filosofía que ya animara el primer Plan de Convergencia: desplazamiento hacia el futuro de los mayores esfuerzos de contención del gasto y el déficit y una excesiva confianza en el crecimiento económico como motor del ajuste.

Uno de los compromisos incluidos en el segundo Plan de Convergencia era el de reformar la Ley General Presupuestaria, en un reconocimiento implícito de la importancia de las instituciones y los procesos presupuestarios para garantizar la disciplina fiscal, la capacidad estabilizadora del presupuesto y su sostenibilidad a medio y largo plazo. Si en algo hay que valorar los resultados de la experiencia comparada y de la propia sucintamente presentados en este trabajo, sólo cabe esperar que la anunciada reforma contribuya positivamente a la disciplina si consigue eliminar la influencia de las principales causas de la actual laxitud: incrementalismo arraigado, excesivo peso estratégico de los ministros de gasto, escasa integración y carencia de dirección estratégica de los procesos de elaboración y gestión y extraordinaria flexibilidad en la ejecución, rasgo éste que mina el interés y la capacidad de resolución de conflictos de los participantes en la elaboración y la discusión parlamentaria. Sobre la base de un avance real en este terreno, el sector público espanol podría, por fin, disponerse a afrontar el gran reto de fin de siglo: la ya largamente pospuesta modernización de la administración pública, basada en criterios de responsabilidad, minimización de costes y atención al usuario.

\section{Notas}

- Catedrático y Director del Departamento de Hacienda Pública de la Universidad Complutense de Madrid. Director del Centro de Estudios sobre Economía Pública de la Fundación BBV.

' Este trabajo, basado parcialmente en ideas desarrolladas en González-Páramo (1995), se ha beneficiado de los comentarios recibidos en el seminario Economía e Instituciones (UIMP, Formigal-Huesca, 20 de septiembre de 1995).

2 Pollard (1993) y Doyle y WEALE (1995) subrayan, no obstante, que el papel de la independencia es menor en periodos de tipos de cambio fijos o estables. Asimismo, la independencia podría justificarse en muchos casos como la plasmación institucional de la aversión social a la inflación, más que como la causa de unas tasas de inflación reducidas.

${ }^{3}$ La tendencia a largo plazo al aumento de la ratio gasto/PIB será difícil de compensar con un incremento en la ratio ingresos/ $\mathrm{PIB}$, especialmente en aquellos paises donde los ingresos constituyen un porcentaje alto del PIB. A medida que aumenta la presión fiscal se tiende a observar una mayor evasión, así como otros efectos adversos sobre los incentivos y la eficiencia económica. En este marco, la consolidación presupuestaria en la mayoría de los países miembros debería centrarse, principalmente, en la reducción del gasto público más que en el aumento de la carga fiscal del sector privado (Instituto Monetario Europeo, 1995). Por otra parte, The Economist (1995) subraya que de los 52 reajustes presupuestarios realizados en los paises de la OCDE entre 1964 y 1992, sólo 14 tuvieron éxito, y, en estos casos, el 80 por 100 de la reducción del déficit provino de recontes del gasto.

- Los principales trabajos empíricos en esta materia son los de GriLl y otros (1991), Alesina y Tabellini (1990), Alesina y Drazen (1991), RoubinI y SaCHS (1989a, 1989b) y VON HAGEN $(1991,1992)$. Una buena sintesis es la ofrecida por ALESINA Y PEROTT (1995).

' Incluso al nivel más primario, que es el estadístico, la relación de interdependencia entre gastos e ingresos públicos tiende a presentar diferencias entre países. En el caso de España, por ejemplo, la dinámica presupuestaria parece estar dominada por los ingresos públicos: los aumentos de presión fiscal sólo son eficaces transitoriamente y tienden a generar un mayor déficit en el futuro. En otros países el patrón de interdependencia gastos-ingresos presenta rasgos distintos (véase Gonzálzz-Páramo, 1994, y las referencias en éste citadas).

6 El contenido de estas secciones se basa en González-Páramo (1995).

' VON HAGEN y HARDEN (1994) hacen notar que la primera estrategia tiende a ser adoptada por países "grandes", con administraciones públicas complejas y una heterogeneidad apreciable de los intereses de los participantes en el proceso presupuestario.
${ }^{8}$ La construcción de los indices se detalla en Von HaGen (1992). En la figura 2 aparecen representados en porcentaje del máximo valor alcanzable en cada caso. Hemos excluido a Luxemburgo a causa de lo incompleto de la información disponible.

9 Von HaGen y HaRden (1994) encuentran un nexo empírico muy significativo: instituciones presupuestarias-proporción de gastos sin tope estricto (ampliables y suplementarios) y entre éstos y una pobre posición de las finanzas públicas en materia de sostenibilidad a largo plazo.

${ }^{10}$ HarRison (1991) ofrece un interesante y detallado análisis crítico de la experiencia británica a lo largo de la década 1979-1989. En OCDE (1990 y 1991) se contiene abundante información sobre las experiencias de gestión pública llevadas a cabo en los paises miembros. OxIEY (1993) describe y valora algunas de estas experiencias. OrTúN (1993) es una buena referencia sobre los conceptos básicos en materia de gestión pública.

"Para una descripción y una valoración de los cambios introducidos, pueden consultarse los trabajos de OXIEY (1993), Easton (1995) y Holtham y Kay (1995).

${ }^{12}$ Un caso que ha suscitado gran atención internacional ha sido la regulación que desde febrero de 1990 rige la actuación del Banco de la Reserva de Nueva Zelanda. Antes de nombrarse gobemador del Banco, el candidato y el ministro de Hacienda negocian objetivos a alcanzar en términos de algún nivel de precios. El gobierno puede relevar al gobernador de su cargo si los objetivos no se cumplen por razones distintas de las previstas en el propio contrato. Las características de transparencia y responsabilidad de este diseno no deben confundirse con la independencia del banco emisor en el sentido que puede tener este término en el caso del Bundesbank. El gobierno puede tomar las riendas de la politica monetaria durante seis meses a un ciento coste, y los objetivos son fijados por el gobierno. El banco es el agente y el gobierno el principal.

13 Todos los Estados, excepto Vermont, tienen una de las dos limitaciones, con grados diferentes de rigor en su aplicación (ANDERSON y ACIR, 1987).

${ }^{14}$ La experiencia norteamericana es también ilustrativa. Como indica el relativo éxito de la Budget Enforcement Act del presidente Bush, buena parte del rotundo fracaso de la enmienda Gramm-Rudman-Hollings en conseguir sus objetivos cuantitativos puede atribuirse a un defectuoso procedimiento, que no protegía los acuerdos presupuestarios entre el presidente y el congreso frente a ulteriores modificaciones en el propio congreso.

is Puede encontrarse una exposición más detallada de los principales rasgos del modelo presupuestario español en González-Páramo, Utrilla y Vauño (1994), RUIZ-HUERTA Y GIMÉNEZ (1993) y VON HAGEN Y HARDEN (1994). 
- Las medidas de restricción presupuestaria basadas en aumentos de presión fiscal o en la contención de la inversión pública sólo tienen efectos transitorios sobre el déficit, pero sus consecuencias negativas sobre la productividad y el crecimiento económico tienen carácter permanente. Los recontes de la inversión pública tienden a sustituirse con rapidez por ouros gastos, mientras que los aumentos impositivos traen como resultado un relajamiento de la restricción del gasto en lugar de unas menores necesidades públicas de financiación (véase GonZÁlEZ-Píramo, 1994).

" Un detallado estudio sobre el presupuesto como instrumento de control en Esparia puede encontrarse en EDO, DE PABLOS y VALNío (1994).

${ }^{18}$ La ejecución presupuestaria de 1994 no rompería con este modelo, pues no lo pretendía. El reto fundamental de los presupuestos de 1994 -una vez reconocida la inviabilidad del primer Plan de Convergencia- era relativa- mente modesto: cumplir el objetivo de déficit del 6,4 por 100 para el conjunto de las AA.PP. en un marco de bajo crecimiento (1,3 por 100). En julio, con motivo de la aprobación del segundo Plan de Convergencia, este objetivo fue revisado al 6,7 por 100 debido a un mayor déficit imputado a las administraciones territoriales. Utilizando criterios contables homogéneos, las cifras definitivas de 1994 habrán sobrepasado el objetivo revisado en 0,3 puntos del PIB, en un contexto de crecimiento económico superior al proyectado. Así, pese a la orientación restrictiva de los presupuestos de 1994, a falta de otra "tecnología de compromiso" que el del cumplimiento de un objetivo cuantitativo, puede afirmarse que estos resultados suponen un avance cono en la corrección del déficit. Conto en cuanto al nivel de partida del déficit estructural -que la OCDE y la UE estiman entre el 5 y el 6 por 100 del PIB- y corto respecto de la necesaria disminución del ritmo de acumulación de la deuda pública.

\section{Bibliografia}

Alesina, A., y Tabeluni, G. (1990): "A positive theory of fiscal deficits and government debt", Review of Economic Studies, 57.

Alesina, A., y Drazen, A. (1991): "Why are stabilizations delayed? A political economy model", American Economic Review, 81.

ANDERSON, G., y ACIR (US Advisory Commission on Intergovernmental Relations) (1987): Fiscal discipline in the federal system: National reform and the experience of the States, ACIR, Washington.

ANDRÉS, J.; BosCÁ, J. E., y DOMÉNECH, R. (1995): "Main patterns of economic growth in OECD countries", Investigaciones Económicas, XIX, enero.

BayOumi, T., y EICHENGREeN, B. (1995): "Restraining yourself: The implications of fiscal rules for economic stabilization", IMF Staff Papers, 42.

Bean, C. (1992): "Economic and Monetary Union in Europe", Centre for Economic Performance, Discussion Paper, núm. 86, julio.

Blchanan, J., y WaGner, R. (1977): Democracy in deficit, Academic Press, New York. BuIter, W. (1992): "Should we worry about the fiscal numerology of Maastricht", CEPR, Discussion Paper Series, 668, junio.

BUTTRR, W.; CORSETT1, G., y ROUBIN, N. (1992): "Excessive deficits': Sense and nonsense in the Treaty of Maastricht", CEPR, Discussion Paper Series, 750, diciembre.

Comisión CE (1991): "One market, one money", European Economy, 44.

Comite Delors (1989): "Report on Economic and Monetary Union in the European Community", Comisión de la CE, Bruselas.

Cukierman, A. (1992): Central bank strategy, credibility, and independence, MIT Press, Cambridge, Massachusetts.

De HaAn, J., y StURm, J. E. (1994): "Political and institutional determinants of fiscal policy in the European Community", Public Cboice, 80.

DE LonG, J. B., y SummfrRs, L. H. (1992): "Macroeconomic policy and long-run growth", Federal Reserve Bank of Kansas City, Economic Review, 77.

DOYLE, C., y WEALE, M. (1995): "Do we really want and independent central bank", Oxford Review of Economic Policy, 10.

EASTON, B. (1995): "Economic and other ideas behind the New Zealand reforms", Oxford Review of Economic Policy, 10.

EDO, V.; DE PABLOS, L., y VALiNo, A. (1994): El presupuesto como instrumento de control del gasto público, Fundación BBV (Centro de Estudios sobre Economía Pública), Documentos de Trabajo, Bilbao.

FISCHER, S. (1991): "Growth, macroeconomics, and development", NBER, Working Paper, 3702.

Fuentes Quintana, E. (1985): "Hacienda pública y déficit", Papeles de Economia Española, 24.
Gonzalez-Páramo, J. M. (1994): “'Tax and spend' or 'spend and tax'? Causality between revenues and expenditures and the size of the public budget in Spain, 1955-1991 ${ }^{n}$, European Economy, Reports and Studies, 3.

Gonzálzz-Páramo, J. M. (1995): "Las vías de la disciplina presupuestaria: Opciones y experiencia comparada", Fundación FIES-CECA, Cuadernos de Información Económica, 100, julio.

GonzÁlez-Páramo, J. M.; Utrila, A., y Valiño, A. (1994): "Fiscal policy in Spain, 1980-1992: main developments and current issues", European Economy, Reports and Studies, 3.

Grum, V.; Masclandiaro, D., y TABeunN, G. (1991): "Political and monetary institutions and public finance policies in the industrial democracies" Economic Policy, 13.

Grimes, A. (1991): "The effects of inflation on growth: some international evidence", Weltwirtschaftlicbes Archiv, 127.

Gros, D. (1991): "La UEM y la política fiscal", Papeles de Economia Española, 48. HARRISON, A. (1991): El control del gasto público (1979-1989), Instituto de Estudios Fiscales, Ministerio de Economía y Hacienda, Madrid.

Holtham, G., y KaY, J. (1995): "The assessment: institutions of policy", Oxford Review of Economic Policy, 10.

INSTTUTO MONFTARIO EUROPEO (1995): Informe Anual 1994, Frankfort, abril.

Kormendi, R., y MeGuIRE, P. (1985): "Macroeconomic determinants of growth. Cross-country evidence", Jourmal of Monetary Economics, 16.

OCDE (1990): Public management developments. Survey 1990, París.

OCDE (1991): Public management developments. Update 1991, París.

ORTúN, V. (1993): Gestión püblica, Fundación Banco Bilbao Vizcaya, Programa de Estudios sobre Economía Pública, Documentos de Trabajo.

OxaEy, H. (1993): El sector público en los 80 y experimentos de política puiblica para los 90, Fundación Banco Bilbao Vizcaya, Programa de Estudios sobre Economía del Sector Público, Documentos de Trabajo.

POLARD, P. (1993): "Central bank independence and economic performance", Federal Reserve Bank of St. Louis Review, 75, julio-agosto, 21-36.

RAYMOND, J. L. (1993): "Acortamiento de distancias, convergencia y competitividad en los paises de la Europa de los Doce", Papeles de Economia Española, 56.

RUIZ-HUERTA, J., y GIMÉNEZ, A. (1993) (eds): Estructura instilucional y gestión del gasto püblico en algunos paises de la OCDE, Instituto de Estudios Fiscales, Ministerio de Economía y Hacienda, Madrid.

RouBiN, N., y SACHS, J. (1989a): "Political and economic determinants of budget deficits in the industrial democracies", European Economic Review, 33. 


\section{Bibliografía}

RouBIN, N., y SACHS, J. (1989b): "Government spending and budget deficits in the industrial countries", Economic Policy, 8.

SAmuElson, R. J. (1988): "Backdoor govemment", Newsweek, 62, 17 de octubre. The Economist (1995): "Europe's totem pole", 22-29 de septiembre.

VON HAGEN, J. (1991): "A note on the empirical effectiveness of formal fiscal restraints", Joumal of Public Economics, 44.

VON HAGEN, J. (1992): "Budgeting procedures and fiscal performance in the European Communities", EEC Commission, Economic Papers, 96, octubre.
VON HAGEN, J., y HARDEN, I. (1994): "National budget processes and fiscal performance", European Economy, Reports and Studies, 3.

VON HAGEN, J., y HARDEN, I. (1995): "Budget processes and commitment to fiscal discipline", European Economic Review, 39.

WiLDavsky, A. (1986): "La teoría de la limitación del gasto", Papeles de Economia Española, 27.

WILuAmson, O. (1993): "The economic analysis of institutions and organisations", Working Papers, 133, OECD, París. 